\title{
Spine Crossing Minimization in Upward Topological Book Embeddings
}

\author{
Tamara Mchedlidze and Antonios Symvonis \\ Dept. of Mathematics, National Technical University of Athens, Athens, Greece \\ \{mchet, symvonis\}@math.ntua.gr
}

\section{Introduction}

An upward topological book embedding of a planar st-digraph $G$ is an upward planar drawing of $G$ such that its vertices are aligned along the vertical line, called the spine, and each edge is represented as a simple Jordan curve which is divided by the intersections with the spine (spine crossings) into segments such that any two consecutive segments are located at opposite sides of the spine. When we treat the problem of obtaining an upward topological book embedding as an optimization problem, we are naturally interested in embeddings with the minimum possible number of spine crossing.

We define the problem of HP-completion with crossing minimization problem (for short, $H P C C M$ ) as follows: Given an embedded planar graph $G=(V, E)$, directed or undirected, one non-negative integer $c$, and two vertices $s, t \in V$, the HPCCM problem asks whether there exists a superset $E^{\prime}$ containing $E$ and a drawing $\Gamma\left(G^{\prime}\right)$ of graph $G^{\prime}=\left(V, E^{\prime}\right)$ such that (i) $G^{\prime}$ has a hamiltonian path from vertex $s$ to vertex $t$, (ii) $\Gamma\left(G^{\prime}\right)$ has at most $c$ edge crossings, and (iii) $\Gamma\left(G^{\prime}\right)$ preserves the embedded planar graph $G$. When the input digraph $G$ is acyclic, we can insist on HP-completion sets which leave the HP-completed digraph $G^{\prime}$ also acyclic. We refer to this version of the problem as the Acyclic-HPCCM problem.

\section{Results}

A detailed presentation of our results (including technical proofs) is available as a Technical Report through arXiv [1. The following theorem establishes the equivalence between the Acyclic-HPCCM problem and the problem of spine crossing minimization in upward topological book embedding for st-digraphs.

Theorem 1. Let $G=(V, E)$ be an $n$ node st-digraph. $G$ has a crossing-optimal $H P$-completion set $E_{c}$ with Hamiltonian path $P=\left(s=v_{1}, v_{2}, \ldots, v_{n}=t\right)$ such that the corresponding optimal drawing $\Gamma\left(G^{\prime}\right)$ of $G^{\prime}=\left(V, E \cup E_{c}\right)$ has c crossings if and only if $G$ has an optimal (wrt the number of spine crossings) upward topological book embedding with $c$ spine crossings where the vertices appear on the spine in the order $\Pi=\left(s=v_{1}, v_{2}, \ldots, v_{n}=t\right)$. 


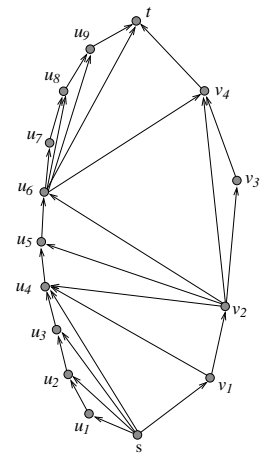

(a)

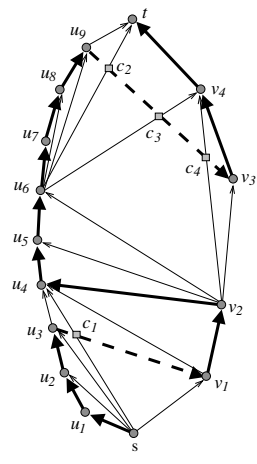

(b)

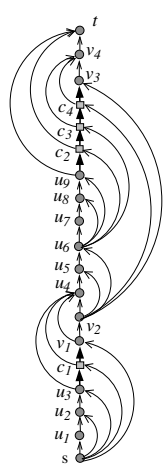

(c)

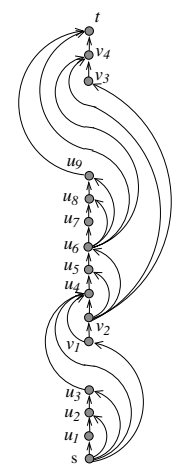

(d)

Fig. 1. Example of the construction of an optimal topological book embedding

For an outerplanar triangulated st-digraph $G$, we define the st-polygon decomposition of $G$ and, based on the decomposition's properties, we develop a lineartime dynamic programming algorithm that solves the Acyclic-HPCCM problem with at most one crossing per edge. This is summarized in the following theorem.

Theorem 2. Given an $n$ node outerplanar triangulated st-digraph $G, a$ crossing-optimal HP-completion set for $G$ with at most one crossing per edge can be computed in $O(n)$ time.

Our main result follows from the Theorems $1 \& 2$ :

Theorem 3. Given an n node outerplanar triangulated st-digraph $G$, an upward topological book embedding for $G$ with minimum number of spine crossings and at most one spine crossing per edge can be computed in $O(n)$ time.

Figure 1] a shows an upward planar st-digraph $G$ that is not hamiltonian. In Figure 1.b graph $G$ is augmented by the edges of an optimal HP-completion set (bold dashed edges) produced by our algorithm. The created Hamiltonian path is drawn with bold edges. By splitting the crossing edges we obtain graph $G_{c}$. Figure 1. shows an upward topological book embedding of $G_{c}$ with its vertices placed on the spine in the order they appear on a hamiltonian path of $G_{c}$. The edges appearing on the left (resp. right) side of the Hamiltonian path (as traveling from $s$ to $t$ ) are placed on the left (resp. right) half-plane. Figure 1 d shows the optimal upward topological book embedding of $G$ created from the drawing in Figure 1]c by deleting $c_{1}, c_{2}, c_{3}, c_{4}$ and merging the split edges of $G$.

\section{References}

1. Mchedlidze, T., Symvonis, A.: Optimal acyclic hamiltonian path completion for outerplanar triangulated st-digraphs (with application to upward topological book embeddings). arXiv:0807.2330, http://arxiv.org/abs/0807.2330 